\title{
Graphemic analysis underlying literacy
}

\author{
PAUL A. KOLERS \\ University of Toronto, Toronto, Ontario MSS IAI, Canada \\ SANDRA R. PALEF \\ Ontario Institute for Studies in Education, Toronto, Ontario M5S IV6, Canada \\ and \\ LEW B. STELMACH \\ University of Toronto, Toronto, Ontario M5S 1A1, Canada
}

\begin{abstract}
Three experiments are described that studied the role of detailed graphemic analysis upon the ability to read text. College students named letters in various approximations to English, with frequency of individual letters constant. Findings were that later skill at reading varied with the order of approximation to English of the letters that were named, that the spacing of the letter sequences was important to this result, and, finally, that the influence of specific visual practice extended to the typeface on which the naming and reading were carried out. Hence, rather than a letter-by-letter process or its opposite, a wholly semantic analysis, reading is shown to be intimately dependent upon details of visual analysis of patterns or letter sequences.
\end{abstract}

Contemporary theory accounting for the recognition of words and sentences usually makes a sharp distinction between the acquisitive operations directed at a visual or auditory stimulus and its representation in memory. In the widely maintained distinction between the operations of short-term and of long-term memory, kind of operation is sometimes identified with kind of memory. Generally, the chief operation is thought to be extraction of its gist or meaning from a verbal stimulus in short-term memory and comparison of the gist to others during its transfer for permanent rest to long-term memory. For convenience, this proposal can be referred to as a two-stage theory of analysis and comparison (Kolers \& Perkins, 1975).

A useful contrast to two-stage theories is the proposal that people encode events in all the ways they can, consistent with their purpose and the time available, and that the analyses carried out are the representation of the event. Recognition is not matching a sample to some fixed template or formula stored in mind, but is due to a facilitated execution of encoding activities. Memory, in this view, is not a place where items are stored, but more a matter of manifesting skills in encounters with objects (Kolers, 1979).

This work was supported by Grant A7655 from the Natural Sciences and Engineering Research Council of Canada to the first author. We thank Richard Wolfe for devising the program that generated the statistical approximations and Joan Cherry for testing the subjects in Experiment 2. Address of the first author is P. A. Kolers, Department of Psychology, University of Toronto, Toronto, Ontario, Canada M5S 1A1.
Studying recognition of verbal materials provides a way to make some of these assertions more concrete, and the present paper describes three experiments for that purpose. The contrast between two-stage theories and the present view may be put as between recognition of words as due principally to recognition of their semantic features vs. recognition of words as due principally to the analytical operations carried out upon them.

It could be argued that such a test is properly carried out only on typographically and orthographically normal material. As has been stated before, however, the skilled reader is so skilled that many components of performance, the variables of interest, are often obscured when normal text is used (Kolers \& Perkins, 1975). In order to slow down the reader and magnify some of the constituents of performance, we use geometrically transformed materials-letters or words that preserve all of the relevant properties of the language except the appearance of the type. Acquisition of skill with these texts and transfer of skill across conditions are thought to tap processes that are similarly tapped by naming letters or reading text in normal typographic arrangement.

\section{EXPERIMENT 1}

Studying the influence of practice at naming letters on reading words, Kolers and Magee (1978) suggested that further tests might profitably manipulate the order of approximation to English of the practice material. The underlying idea is to evaluate practice 
with letter sequences as a variable affecting performance on typographically unfamiliar text. The present experiment therefore required college students, as training, to name individual letters that were in first order or fourth order approximations to English and then, as test, to read connected English prose.

\section{Method}

Materials. Twenty-seven passages of approximately 300 words each were selected from Miller (1962). Fifteen of the passages were used to produce first order and fourth order approximations to English as training materials. For first order approximation, the letters and spaces of each passage were randomized by computer and printed as pseudowords with a maximum length of 10 letters. Fourth order approximations were generated by reading in each letter and space in the text as a series of combinations four units in length. These were recorded by computer and displayed as a text that preserved approximately the letter sequences and word lengths of the original. When a text is used as a source for higher order approximations to English, it is often difficult to maintain complete fidelity to the statistics of the original. Often a shortage of vowels occurs, or other statistical constraints mar the similarity of output text to input text. In the present case, the program was run many times on each page of text until an output was obtained that used all of the combinations of four letter and space units in the original exactly once, and fewer than five extra combinations were required to use all of the letters in the original exactly once. In this way, the literal contents of a page of text were preserved as units of four letters identical to the original but scrambled as to sequence. Thus both a first order and a fourth order transformation that was applied to the same input page presented the individual letters of the original approximately equally, but the fourth order approximation preserved more of the sequence of the original (see Figure 1 for examples).

In both approximations the case of the letters in the original passage, upper- or lowercase, was maintained. Passages were presented in the format of approximately 65 characters and spaces per line and occupied approximately 26 lines each. Ten of the 12 remaining pages of connected discourse were used as test materials. The last two pages were used to produce randomly ordered letters, and a space separated each letter from the next one on a line.

All of the material was typed in IBM Courier font for Experiments 1 and 2 and in Courier and in italic for Experiment 3. All of the materials were inverted geometrically for presentation.

Subjects. Two groups of 10 subjects each were composed of university students or recent graduates. All were right-handed native users of English. Their reading skills were restricted to languages using the Roman alphabet. They were all able to read aloud a criterion page of about 300 words in less than $2 \mathrm{~min}$. They were paid for participating in the study.

Procedure. Subjects were placed alternately according to arrival in the group practiced with first order or fourth order texts. After reading the criterion page, the subjects were instructed to name pages of letters aloud as rapidly as they could accurately; they were allowed a 1 -min rest after each page. The pages were held by a reading stand about 15 in. from the subject's eyes, and the person was not allowed to point, squint, move the head, or the like. The reading was recorded on tape and timed by stopwatch. Subjects were tested individually. They named the letters or read as many pages as they could in $1 \mathrm{~h}$. The subjects then returned on successive days until they had named all 15 passages of letters, read 10 passages of text, and named the letters of the final two pages of scrambled letters. The last-named task always occurred after reading at least two pages of inverted text on the final test day. Between five and seven sessions were required for each subject tested.
For ease of description we refer to initial letter naming as training, subsequent reading of text as test, and final letter naming as naming scrambled or spaced letters. In Experiment 1 all the training, test, and spaced letters passages were in IBM Courier type only.

\section{Results}

Figure 2 shows the speed with which letters were named during training, text was reading during test, and spaced letters were named, all on arithmetic coordinates. Because means and variances were correlated, the data were transformed to logarithms, shown in Figure 3, and analysis of variance was carried out on the transformed scores. Speed of naming letters increased rapidly during the training phase for both orders of approximation but was greater for fourth order than for first order; the rate of improvement was about the same for both orders, however. There was improvement with successive pages $[\mathrm{F}(14,252)=90.27, \mathrm{p}<$ $.01]$ and the two orders differed in speed $[F(1,18)=$ $8.51, \mathrm{p}<.01]$; the interaction of Order by Pages (comparing the slopes of the lines) was nonsignificant $[F(14,252)=1.80, p>.05]$. Hence, the more closely the letters approximated to English orthography, the more rapidly they were named.

Speed of reading text is shown in the test phase of the figure. Practice at naming the first order letters transferred well to reading text, but transfer from fourth order letters was even better. The difference between the two test performances was significant $[F(1,18)=11.41]$; the effect of practice at reading the 10 pages of text was also marked $[F(9,162)=29.11]$; and the slopes of the lines were different, as measured by the interaction of Order of Approximation by Page Number $[F(9,162)=3.70$, all ps $<.01]$. Hence, practice at naming letters transferred positively to reading text, and degree of transfer was greater the more closely the letter sequences approximated to the orthography of normal English.

In Phase 3 of the study, subjects named two pages of letters that were in first order approximation to English, with the individual letters separated by spaces. The difference in time between the first and second pages was significant $[F(1,18)=10.34, p<.01]$, but the difference between subjects trained on the first and on the fourth order approximations was not significant $[F(1,18)<1]$.

\section{Discussion}

Letters whose sequence approximated more closely to the spelling pattern of words were named more rapidly than were others, and practice at naming letters in the relevant sequence provided more skill for subsequent reading of text. Neither naming these letters nor reading the text provided much training for subsequent naming of individual letters.

One account of the results on initial naming might suppose that the subjects were able to use their knowledge of English, and, as fourth order approximations 


\section{ITALIC 1St ORDER}

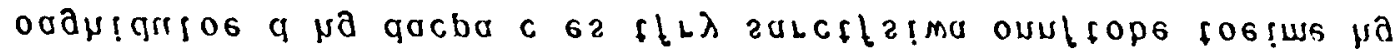

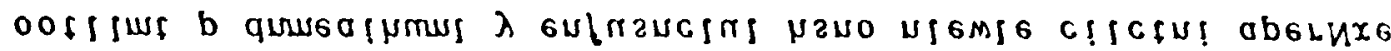

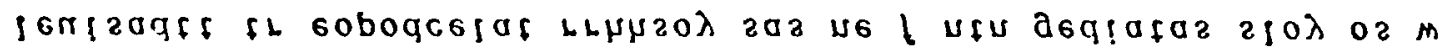

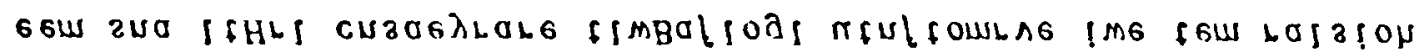

\section{COURIER ISt ORDER}

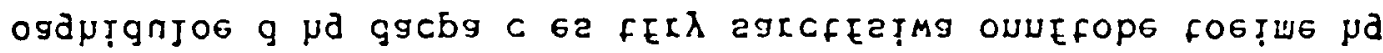

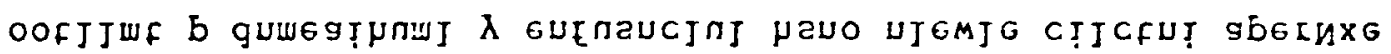

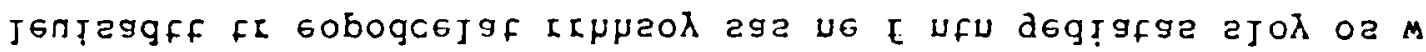

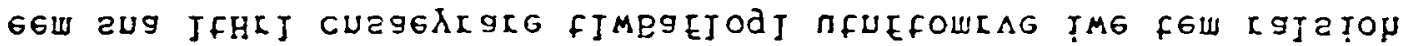

\section{ITALIC 4th ORDER}

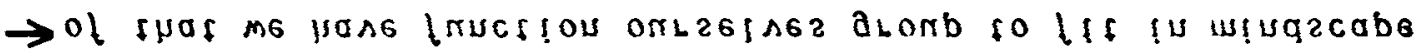

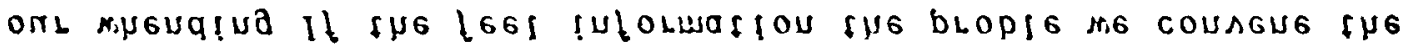

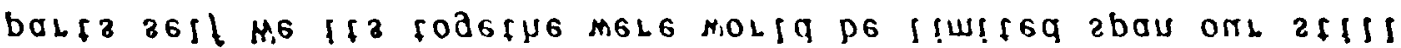

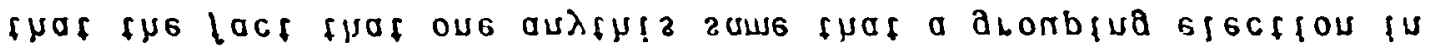

\section{COURIER 4th ORDER}

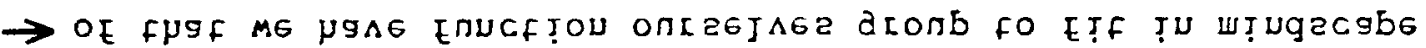

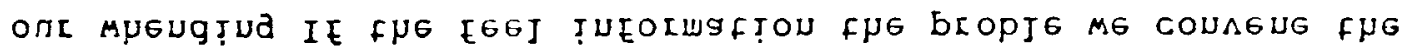

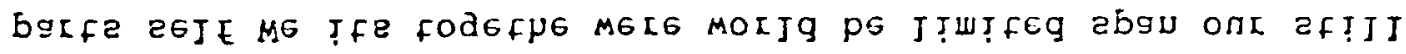

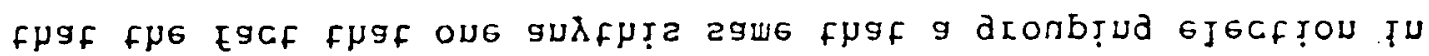

Figure 1. Examples of text. Experiments 1 and 2 used only the texts in Courier typeface, but all four examples were used in Experiment 3.

came closer to words than did first order approximations, the subjects were able to profit from preview or anticipation of fourth order sequences more than the first order sequences. Practice with the sequences as syllables or words would, in turn, aid reading in the test phase. Aspects of this view were tested in Experiments 2 and 3 and found wanting. An alternative is that exposure to and practice in analyzing sequences of letters forms the basis of the performance.

The influence of practice with different orders of approximation argues that the effect of exposure and analysis is not chiefly to aid the encoding of individual letters as, say, Mason (1975) or Massaro (1979) might contend, but rather to aid analysis of sequences. It is well known, of course, that sequences that preserve structural properties of a language aid its processing, in memory (Miller, Bruner, \& Postman, 1954), in reading strings of words aloud (Kolers, 1969), and in other tasks. This influence of sequence argues for specificity or variability of object analysis in recognition, rather than for recognition of elements composing the objects. That is, it argues for recognition of letters, not their 


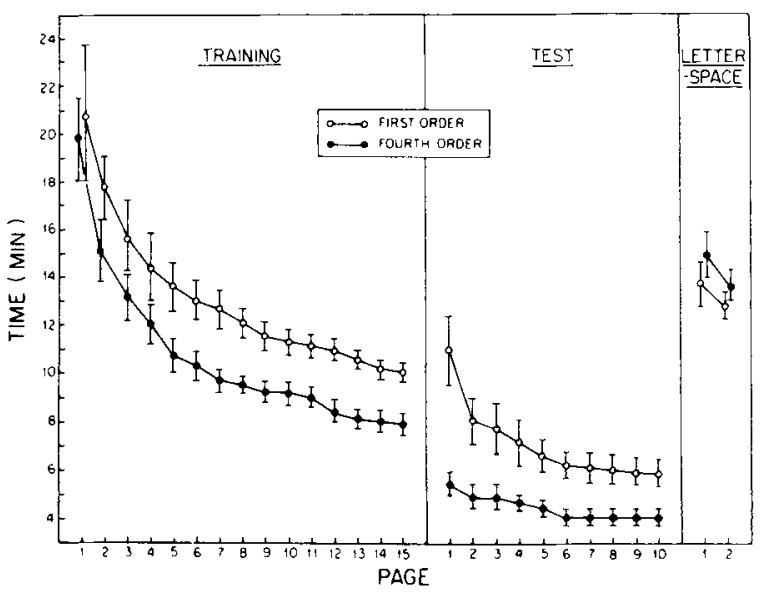

Figure 2. Results of Experiment 1. The separate phases of the study are shown as training (naming letters), test (reading), and a further naming of letters. Vertical bars mark \pm 1 standard error.

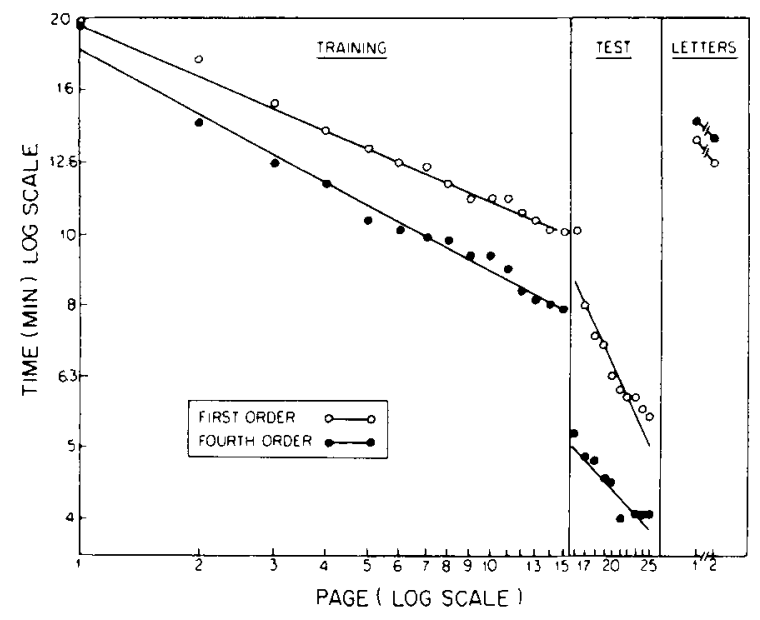

Figure 3. The results of Experiment 1 shown on logarithmic coordinates. The lines are least-squares fits.

features, when letters must be recognized and for letter sequences, not letters, when sequences are required, as follows.

The subjects required $20.4 \mathrm{~min}$ to name the first page of letters during training (combined average) and only 14.2 min to name the first page of scrambled letters, a savings equal roughly to three to four pages of practice at naming letters as pseudowords (Figure 2). The two training tasks had about the same influence on subsequent ability to name isolated scrambled letters, moreover. The contrast with the results found by Kolers and Magee (1978) is marked. Their subjects either named letters or read text as practice for later reading or letter naming. The people trained on naming letters preserved that skill across an intervening period of reading. In the present study a clear decrement was found between the end of training on naming letters aggregated as pseudowords, either in first or fourth order approximations, and later naming of first order letters separated by spaces. A very important difference between the two studies is that the Kolers and Magee (1978) study trained subjects on passages of letters that were separated, each letter followed by a blank space; in the present study the training letters were aggregated into pseudowords. So we found that practice at naming letters that are assembled into sequences transfers only moderately to naming individualized letters.

These findings suggest that the precise spatial organization of letter sequences within the demands of the task plays a significant role in recognitive procedures. The suggestion is tested in the following study.

\section{EXPERIMENT 2}

Aggregations of letters are different as visual patterns from dispersed letters, their orders of approximation to English notwithstanding. In our conjecture it is the letters as a visible pattern, as much as their orthography, that accounts for the results obtained. Hence, a reasonable question concerns the influence upon performance of spacing the letter sequences. The present experiment compared the influence on subsequent reading of text of training on naming first and fourth order approximations to English, as in Experiment 1, but with the letters of the training pages each separated by a space.

\section{Method}

The same texts were used in Experiments 1 and 2, except that the sequences in Experiment 2 were arrayed as 26 lines of 32 letters per line, each letter after the first on a line separated from the preceding letter by a space. The subjects named 15 pages of letters, arranged as first or as fourth order approximations to English, and then read 10 pages of geometrically inverted text. Fourteen undergraduates at the University of Toronto were tested, seven for each approximation; the testing procedures were much the same as those in Experiment 1.

\section{Results}

Figure 4 shows the learning curves for the two conditions. Analysis of variance of the logarithmically transformed data yielded a significant effect for practice $[F(14,168)=39.03$ and $F(9,108)=33.77$ for

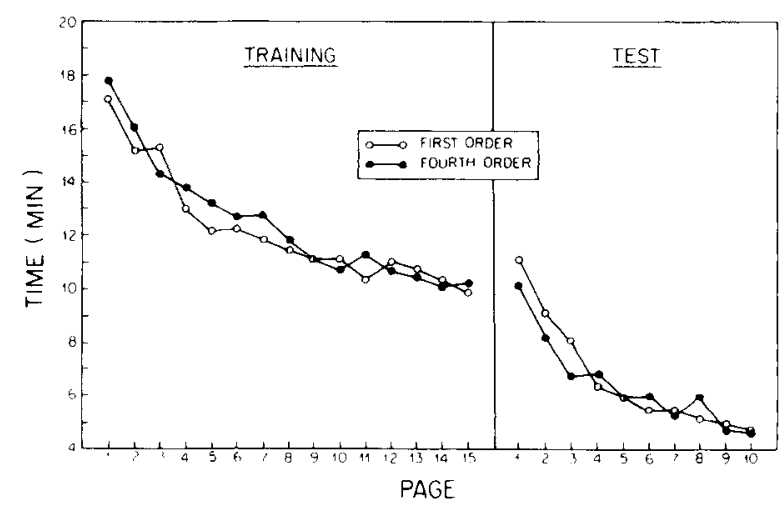

Figure 4. Results of Experiment 2. 
the training and test phases, respectively, $p<.01$ in both cases]. No difference was found between first and fourth order approximations as tested on the raw scores, on their logarithmic transformation, or on their reciprocals ( $F<1$ in all cases), nor for the interaction of Pages by Order of Approximation. Visual inspection reveals a considerable overlap between the present data and the first order approximations of Experiment 1 (Figure 2).

\section{Discussion}

Distributing the sequences of letters spatially seems to interfere with the analytical procedures underlying the facilitation described in Experiment 1. A similar test carried out in The Netherlands yielded similarly inconclusive results for various orders of approximation to Dutch (Timmers, 1976). One view of this result emphasizes clustering or unitizing of the sequences, particularly since Mewhort (1966), among others, has shown that detection of words may be influenced by spacing of letters. It does seem plausible that a word boundary that is marked physically would enhance perceiving the bounded items as wholes (as well as lessening discriminability of their components). Though bounding may be an aid, it clearly is not the whole story; Kolers and Lewis (1972, Figure 1) found that familiar words could often be distributed in geometrically unfamiliar configurations without noticeable loss of perceptibility. Several factors are active here. A method for disentangling them that might distinguish between the automatized recognition of sequence and of letter identity uses a writing system that encodes position redundantly. Arabic, for example, uses a different shape depending upon whether a letter is initial, medial, or final. We could use this principle of the Arabic alphabet to code spatial or phonological sequence, by making letters spell a word in the sequence they are written without regard to shape or by making letters spell a word according to shape rather than position. To illustrate, suppose that in English $\mathrm{R}, \mathrm{r},{ }^{*}$ were the three shapes of ar used when the letter begins, is in the middle of, or ends a word; A,a,@ were the same for eh; and T,t, $\$$ were the same for tee. And suppose that the normal spelling were Ra ("rat"). For test purposes we could write *At, preserving word identity by means of sequence while violating shape, or we could write $\$ \mathrm{Ra}$, preserving word identity by means of letter shape while violating sequence. A test that pitted shape information against sequence might help to reveal some of the analytical operations that underlie the execution of skilled analyses of visual patterns.

So far, we have demonstrated that visual exposure to sequences of letters enhances subsequent reading of text in the absence of practice at reading itself but inhibits naming isolated letters. Our assertion is that it is not the linguistic nature of the sequences that underlies the facilitation so much as it is visual experience with them as patterns. The following ex- periment tests this assertion more directly, demonstrating an intimate relation between visual analysis and recognition.

\section{EXPERIMENT 3}

The influence of the linguistic or orthographic component is stressed in many accounts of word recognition, often to the neglect of graphemic properties of the task. In contrast, Kolers and Perkins (1975, Table 3) found that skill at processing a graphemic sequence is often quite specific, and extended practice with one typographic variant was not a sure basis for transfer to other variants, despite identity of the linguistic component across variants. In the present experiment we studied the specificity of the role of the graphemic component in another way and compared it with the orthographic.

\section{Method}

Five pages of letters used in Experiment 1 and three pages of text were prepared for this experiment. The letters, grouped as pseudowords, were typed both in italic and in Courier typeface, and the three pages of text were also in Courier. The two typefaces were combined as training material with two orders of approximation to English, making four training groups (Figure 1). Twelve undergraduates in each group first named the letters of five pages of pseudowords, and then all subjects read the same three pages of text.

\section{Results}

Figure 5 shows the training (naming letters) and test (reading) scores for the four groups of subjects, in arithmetic units. Figure 6 displays the logarithmic transformation of the scores. Analysis of variance of the logarithmically transformed data yielded a significant difference in speed of naming letters as a function of order of approximation to English $[\mathrm{F}(1,44=5.41$, $.01<\mathrm{p}<.05]$, consistent with, although not as strong as, the result found in Experiment 1; a nonsignificant influence of typeface upon speed (italic vs. Courier) $[F(1,44)=2.87, p>05] ;$ a significant effect of practice $[F(4,176)=155.01, p<.01]$; and a significant interaction of Practice by Order of Approximation $[F(4,176)=3.35, p<.01]$. All other interactions

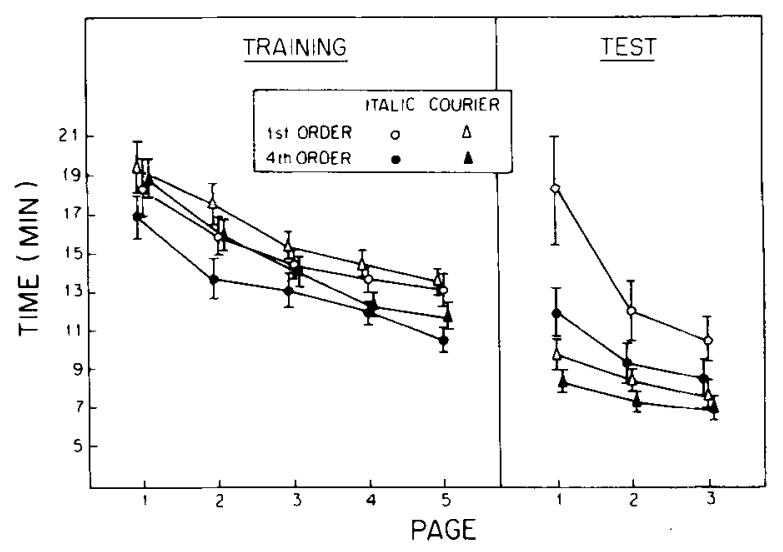

Figure 5. Results of Experiment 3 on arithmetic coordinates. 


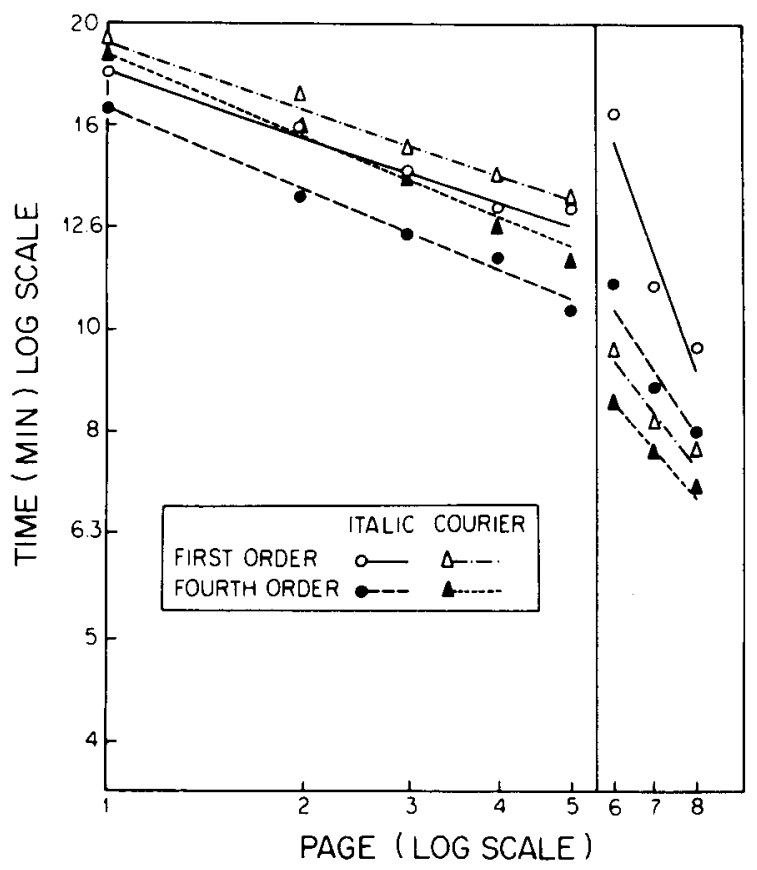

Figure 6. Experiment 3 expressed on logarithmic coordinates. The lines are least-squares fits.

yielded $F<1$. These results on naming letters in different orders of approximation are consistent with those found in Experiment 1, and the two typefaces, if not decisively favoring italic as easier to name, point in that direction.

The results are different for reading. Speed of reading text in Courier typeface did not vary significantly with order of approximation of the training pages $[F(1,44)=3.13, p>.05]$ but did vary with typeface of the training pages $[F(1,44)=6.53, .01<p<.05]$; moreover, the interaction of Page Number by Order of Approximation $[\mathrm{F}(2,88)=4.48]$ and Page Number by Typeface $[F(2,88)=14.85]$ were both significant. (The triple interaction yielded $\mathrm{p}>$.05.) Of greater interest than absolute scores is the transfer of skill from training to test. Transfer scores were calculated by the formula, percentage transfer $=100(\mathrm{~A}-\mathrm{X}) /(\mathrm{A}-\mathrm{B})$, where $A$ is the time to name the first page of letters, $B$ is the time to name the fifth page of letters, and $\mathrm{X}$ is the time to read the first page of text. The effects on transfer of the order of approximation of training pages were nonsignificant $[F(1,44)=1.11$ in arithmetic units and $F<1$ on logarithmic scores], as was the interaction of Order of Approximation by Typeface. The main effect of typeface of training group was significant, however $[F(1,44)=4.38$ on the raw scores and 4.91 on logarithms, $.01<\mathrm{p}<.05$ in both cases] . Hence the approximation to English was less influential on speed of reading than was the typeface of the training and test pages.

\section{GENERAL DISCUSSION}

The chief demonstration of these experiments is the intimate relation between visual analysis of printed words and their recognition. Experiment 1 revealed that letter naming provided more practice for subsequent reading, the more closely the sequences of letters approximated to the normal orthography of English. Experiment 2 showed that the facilitation depended upon the spatial proximity of the letters: Identical letter sequences were less effective when they were spaced out and eliminated word boundaries. Experiment 3 showed in turn that the geometry of the letters, as expressed by their typeface, affected performance and, even with the modest difference in typefaces that we used, affected performance more than did approximation to English. Even within the common task of naming letters, practice at naming letters aggregated into words transferred only to naming separated letters.

It is important to note that the result in Experiment 3 is not a matter of legibility of type as measured, say, by Tinker (1963). The italic used in Experiment 3 was, if anything, somewhat more identifiable than the Courier (Figure 5). Rather, it is the transfer of skill acquired in naming the letters of one typography to skill in reading it that is of interest. All other factors being the same, practice at naming the letters of Courier aided reading Courier more than did practice at naming the letters of italic, despite the small difference in their appearance. It should be realized, of course, that the particular typefaces used were chosen arbitrarily and as a matter of experimenter convenience; moreover, the precise extent of influence of one typography upon another is not the issue of interest. The point of Experiment 3 particularly is to demonstrate that the visual apparatus acquires particular skills with respect to analyzing stimuli, so that practice at processing one typeface transfers less than wholly to performance on another. To put it another way, the point is that a significant part of the recognition of words is tied closely to skill in processing the visual symbols, to graphemic analysis.

These skills can be remarkably specific, even within the domain of a single class of operation. Kolers and Perkins (1975) trained different groups of college students to read some one geometric transformation of English text-inversion, such as was used in the present experiments, rotation, mirror reflection, and others-and then tested subjects' ability to read samples of the other transformations. All of the text was connected English discourse, the samples differing only by virture of the geometric transformation imposed upon them. Percentage transfer from training on one transformation to test on others ranged from $118 \%$ 
(training on inverted text was better practice for reading planar rotation than was training on planar rotation itself) to $3 \%$. A similar specificity is revealed in Experiment 3, in which the degree of transfer from naming letters to reading varied more with the typeface than with the order of approximation to English. Brooks and Miller (1979) also found considerable specificity between training and test, using artificial writing systems for the purpose.

The possible extrapolation from learning to read a novel typography to learning to read a first typography is necessarily limited, but we assume that some principles of pattern recognition are common to the two tasks. Our findings of the specificity in transfer of grouping of characters and in the intimacy of relation between training and test, point to the need for a detailed analysis of the pattern-analyzing operations that mediate these performances.

\section{REFERENCES}

Brooks, L., \& Miller, A. A comparison of explicit and implicit knowledge of an alphabet. In P. A. Kolers, M. E. Wrolstad, \& H. Bouma (Eds.), Processing of visible language 1. New York: Plenum, 1979.

Kole rs, P. A. Clues to a letter's recognition. Journal of Typographic Research, 1969, 3, 145-168.

Kole rS, P. A. Reading and knowing. Canadian Journal of Psychology, 1979, 33, 106-117.
Kolers, P. A., \& LEwis, C. L. Bounding of letter sequences and the integration of visually presented words. Acta Psychologica, 1972, 36, 112-124.

Kolers, P. A., \& MAGEE, L. E. Specificity of pattern-analyzing skills in reading. Canadian Journal of Psychology, 1978, 32, 43-51.

Kolers, P. A., \& Perkins, D. N. Spatial and ordinal components of form perception and literacy. Cognitive Psychology, 1975, 7, 228-267.

MAson, M. Reading ability and letter search time: Effects of orthographic structure defined by single letter positional frequency. Journal of Experimental Psychology: General, 1975, 104, 146-166.

Massaro, D. W. Reading and listening. In P. A. Kolers, M. E. Wrolstad, \& H. Bouma (Eds.), Processing of visible language 1. New York: Plenum, 1979.

Mewhort, D. J. Sequential redundancy and letter spacing as determinants of tachistoscopic recognition. Canadian Journal of Psychology, 1966, 20, 435-444.

Mille R, G. A. Psychology, the science of mental life. New York: Harper \& Row, 1962.

Miller, G. A., Bruner, J. S., \& Postman, L. Familiarity of letter sequences and tachistoscopic identification. Journal of General Psychology, 1954, 50, 129-139.

Timmers, H. Reading geometrically transformed letters and text: Some preliminary results. Annual Progress Report (No. 11). Eindhoven, The Netherlands: Institute for Perception Research (IPO), 1976, pp. 69-75.

TINKER, M. A. Legibility of print. Ames: Iowa State University Press, 1963.

(Received for publication September 26, 1979; revision accepted December 20, 1979.) 